\title{
MODELLING AND SIMULATION OF GREENHOUSE CLIMATE USING DYMOLA
}

\author{
F. Rodríguez ${ }^{*}$, L.J. Yebra ${ }^{\#}$, M. Berenguel ${ }^{*}$, S. Dormido \\ *Universidad de Almería. Dpto. Lenguajes y Computación. Ctra. Sacramento s/n, La \\ Cañada,E04120, Almería, Spain.E-mail: rrodrig@ual.es, beren@ual.es \\ ${ }^{\#}$ CIEMAT-PSA. Apdo. 22, E-04200 Tabernas, Almería, Spain.E-mail:Luis.yebra@psa.es \\ ${ }^{\ddagger}$ U.N.E.D. Facultad de Ciencias. Dpto. de Informática y Automática. Avda. Senda del Rey \\ s/n, E28040, Madrid, Spain. E-mail: sdormido@dia.uned.es
}

\begin{abstract}
This paper deals with the modelling and simulation of greenhouse climate. The obtaining of greenhouse production models (climate, crop development, etc.) is a subject of large interest nowadays, as these models can be used for simulation, control and production optimization purposes. A greenhouse constitutes a complex dynamical system, which is described in this paper using the object-oriented and equation-based declarative modelling language Dymola. A systematic procedure has been followed to allow the development and testing of different submodels, before connecting them to generate the complete greenhouse model. Comparison results between the real and simulated greenhouse are provided. Copyright (C) 2002 IFAC
\end{abstract}

Keywords: Agriculture, object modelling techniques, dynamic modelling, computer simulation, physical models.

\section{INTRODUCTION}

During the last years, a large effort is being devoted in the obtaining of iclimate models of Mediterranean greenhouses, both for simulation and control purposes. This paper deals with the modelling and simulation of a typical Mediterranean greenhouse using the object-oriented and equation-based declarative modelling language Dymola. This model is of capital interest in the study of the dynamics of the greenhouse climate under different control policies, aimed at producing inside climate conditions which helps to avoid extreme situations (high temperature or humidity levels, etc.) and to optimise crop production by achieving adequate temperature integrals while reducing pollution and energy consumption.

The dynamic behaviour of the micro-climate is a combination of physical processes involving energy transfer (radiation and heat) and mass balance (water vapour fluxes and $\mathrm{CO}_{2}$ concentration). These processes depend on the outside environmental conditions, structure of the greenhouse, type and state of the crop and on the effect of the control actuators (typically ventilation and heating to modify inside temperature and humidity conditions, shading and artificial light to change internal radiation, $\mathrm{CO}_{2}$ injection to influence photosynthesis and fogging/cooling for humidity enrichment).
Most of the greenhouse models currently used in the Mediterranean area (made by low cost plastic cover, taking advantage of favourable outside climatic conditions) have been developed empirically and are tailor-made on a hand-craft basis. Some authors of this paper have developed a nonlinear artificial neural network (NN) model of a typical Mediterranean greenhouse (Rodríguez et al., 1999) and a nonlinear model of both climate conditions and crop development using physical laws (Rodríguez et al., 2001). In this paper, the problem of greenhouse climate modelling is treated by following a modular modelling approach. The model is composed by six submodels describing the cover temperature, the soil surface temperature, the first soil layer temperature, the inside air temperature and humidity and the PAR (Photo-synthetically active radiation) radiation. All of them have been developed independently and have been validated using measurements from a real plant sited in Almería (South-East Spain). Once the submodels have been validated, these are connected properly to generate the final compound greenhouse model, which is then simulated and compared again with the experimental data logged from the real plant. As has been mentioned, Dymola is used to describe the structure of the model and its behaviour in terms of differential-algebraic equations (DAE's) with proper language constructs to manage discontinuities by means of implicit events. In addition to the symbolic formula manipulation it 
performs over the DAE system, it gives choice over several known ODE and DAE solvers. In the simulations performed in this work, DASSL (Brenan et. al., 1989), a multistep solver of variable order and step for stiff systems has been selected.

The paper is organised as follows. In $\S 2$, a brief description of a dynamic model of the greenhouse climate is formulated. $\S 3$ is devoted to introduce a model of the greenhouse climate using Dymola. In $\S 4$, some simulation results are shown and compared with real data. Finally, $\S 5$ presents some conclusions.

\section{DYNAMIC MODEL OF GREENHOUSE CLIMATE}

The greenhouse climate can be described by a dynamic model represented by a system of differential equations which can be represented by:

$$
\frac{d X}{d t}=f(X, U, P, V, C, t) \text { with } X\left(t_{i}\right)=X_{i}
$$

$X=X(t)$ is a $\mathrm{n}$-dimensional vector of state variables, $U=U(t)$ is a $\mathrm{m}$-dimensional vector of input variables, $P=P(t)$ is an $o$-dimensional vector of disturbances, $V=V(t)$ is a $p$-dimensional vector of system variables, $C$ is a $q$-dimensional vector of system constants, $t$ is the time, $X_{i}$ is the known initial state at the initial time $t_{i}$ and $f=f(t)$ is a non-linear function based on mass and heat transfer balances.

The number of equations describing the system and their characteristics depend on the greenhouse elements, the installed control actuators and the type of cultivation method. The model presented in this paper corresponds with a typical industrial greenhouse located at the Mediterranean area and has been developed assuming some general hypothesis:

- The greenhouse is divided into four elements: cover, internal air, soil surface and one soil layer. The plants are not considered as an element as no measurements of the leaf temperature is available at the moment, and thus they are considered as a source of disturbances.

a The state variables of the model are the internal air temperature $\left(X_{t a}\right)$ and humidity $\left(X_{h a}\right)$, cover temperature $\left(X_{t c b}\right)$, soil surface temperature $\left(X_{t s s}\right)$ and soil first layer temperature $\left(X_{t s I}\right)$. The PAR radiation onto the canopy (output variable $\left.X_{\text {radPAR,a }}\right)$ is also modelled.

- The disturbance inputs of the system are the outside air temperature $\left(P_{\text {text }}\right)$ and humidity $\left(P_{\text {hext }}\right)$, wind speed $\left(P_{\text {wsext }}\right)$ and direction $\left(P_{\text {wdext }}\right)$, sky temperature $\left(P_{\text {tsky }}\right)$, calculated using the Swinbank formula (Boisson, 1991), deep soil temperature $\left(P_{t d s}\right)$, outside solar radiation $\left(P_{\text {radsol,ext }}\right)$, PAR radiation $\left(P_{\text {radPAR,ext }}\right)$, greenhouse whitening $\left(P_{\text {white }}\right)$ and the evapotranspiration rate inside the greenhouse via the leaf area index $\left(P_{L A I}\right)$.

- The control inputs of the system are the natural ventilation $\left(U_{\text {vent }}\right)$, shade screen $\left(U_{\text {shad }}\right)$ and pipe heating system $\left(U_{\text {heat }}\right)$.

a The heat fluxes are one-dimensional. The model only considers the vertical dimension.
The following physical processes are included in the balances: solar and thermal radiation absorption, heat convection and conduction, crop transpiration, condensation and evaporation. In what follows, a brief description of the models is carried out. A full description can be found in (Rodríguez et al., 2001).

\subsection{Model of the PAR radiation}

The PAR radiation onto the canopy is modelled using a static equation, because it is similar to the PAR radiation outside the greenhouse dimmed by the different physical elements that absorb the radiation (cover material, cover whitening and shade screen).

$$
X_{\text {radPAR, } a}=V_{\text {trs }} \cdot P_{\text {radPAR, ext }}
$$

$V_{t r s}$ is the greenhouse PAR radiation transmission coefficient:

$$
V_{t r s, P A R}=\left\{\begin{array}{cc}
C_{t m, c b} & \text { noshade, nowhitening } \\
C_{t m, c b} * C_{t m . w} & \text { noshade, whitening } \\
C_{t m, c b} * C_{t m . s h a d e} & \text { shade, nowhitening } \\
C_{t m, c b} * C_{t m . w} * C_{t m, \text { shade }} & \text { shade, whitening }
\end{array}\right.
$$

Where $C_{t m, c b}$ is the cover solar transmission coefficient, $C_{t m, w}$ is the whitening solar transmission coefficient and $C_{t m \text {,shade }}$ is the shade screen solar transmission coefficient

\subsection{Heat transfer through the cover.}

The cover of a greenhouse has two sides with different temperatures. Due to the fact that the cover is made using a single material (plastic film) and that it thickness is of a few microns, the conduction heat flux is quantitatively not significant compared with the other fluxes appearing in the balance given in equation (4) (Garzoli and Blackwell, 1981). So, the temperatures of the two sides are assumed to be similar and only one cover temperature has been modelled $\left(X_{t c b}\right)$ using the following heat transfer balance:

$$
\begin{aligned}
& c_{c-e s p, c b} c_{d e n, c b} \frac{c_{v o l, c b}}{c_{a r e a, s}} \frac{d X_{t, c b}}{d t}= \\
& Q_{s o l, c b}-Q_{c v, c b-a}-Q_{c v, c b-e}-Q_{l t, c b}+Q_{r a d, c b}
\end{aligned}
$$

$Q_{s o l, c b}$ is the solar radiation absorbed by the cover, $Q_{c v, c b-e}$ is the convective flux with the outside air, $Q_{c v, c b-a}$ is the convective flux with the internal air, $Q_{l t, c b}$ is the latent heat produced by condensation only on the internal side of the cover, $Q_{\mathrm{rad}, c b}$ is the thermal radiation absorbed by the cover, $c_{c-e s p, c b}$ is the specific heat of the cover material, $c_{d e n, c b}$ is the cover material density, $c_{v o l, c b}$ is the cover volume and $c_{\text {area,s }}$ is the greenhouse soil surface.

\subsection{Heat transfer fluxes in the soil layers}

The soil (greenhouse thermal mass) plays an important role on the greenhouse climate control. During the diurnal time, the soil absorbs the solar radiation on its surface, heating the deep soil layers. During the night, the soil transfers heat to the greenhouse environment from these layers. So the 
conductive fluxes are very significant because this process is the source of the heat fluxes between them. A simple model of the soil has been considered, divided in three layers: surface, first layer and a deep layer with a constant temperature (calculated as the average of the external air temperature during one year). Based on these hypotheses, the temperature of the soil surface (thickness of $5 \mathrm{~cm}$.) is represented by equation (5).

$$
\begin{aligned}
& c_{c-e s p, s s} c_{d e n, s s} c_{e s p, s s} \frac{d X_{t, s s}}{d t}= \\
& =Q_{s o l, s s}-Q_{c v, s s-a}-Q_{c d, s s-s 1}-Q_{l t, s s}+Q_{r a d, s s}
\end{aligned}
$$

where $Q_{\text {sol,ss }}$ is the solar radiation absorbed by the soil surface based on the crop growth status (modelling or measuring the Leaf Area Index-LAI), $Q_{c v, s s-a}$ is the convective flux with the internal air, $Q_{c d, s-s l}$ is the conductive flux between the soil surface and the first soil layer located at $30 \mathrm{~cm}$., $Q_{l t, s s}$ is the latent heat produced by evaporation on the soil surface, $Q_{\text {rad,ss }}$ is the thermal radiation absorbed by the soil surface, $c_{c-e s p, s s}$ is the specific heat of the soil surface material, $c_{d e n, s s}$ is the soil surface material density and $c_{e s p, s s}$ is the thickness of the soil surface.

In the first soil layer, only the conductive fluxes are considered and so, the heat balance in this element is represented by equation (6).

$$
c_{c-e s p, s 1} c_{d e n, s 1} c_{e s p, s 1} \frac{d X_{t, s 1}}{d t}=Q_{c d, s s-s 1}-Q_{c d, s 1-s c}
$$

where $Q_{c d, s s-s I}$ is the conductive flux between the soil surface and the first layer of the soil, $Q_{c d, s l-s c}$ is the conductive flux between the first soil layer and the deep layer at constant temperature, $c_{c-e s p, s l}$ is the specific heat of the first soil layer material, $c_{d e n, s}$ is the first soil layer material density and $c_{e s p, s l}$ is the thickness of this layer.

\subsection{Heat transfer fluxes with the internal air}

The crop affects the greenhouse air temperature. As no measurements of the leaf area are available, it is not possible to use a convective factor in the heat balance equation using it as a boundary variable. The effect of the crop on the air temperature is based on the latent heat due to transpiration of the plants $\left(Q_{t r p}\right)$, using the work of Stanghellini (1987). The cultivation method of the tomato crop used in the installations is NFT (Nutrient Films Technique). The greenhouse contains non-isolated pools in order to recycle the fertilized water to maintain the continuous water flux. The evaporation of the water of the pools affects the greenhouse climate. In the same way the transpiration of the crop has been included in the balances, a new factor has been added to the latent heat term based on the net radiation and the water vapour pressure deficit. Based on all these processes, the greenhouse air temperature can be modelled using the following heat balance equation:

$$
\begin{aligned}
& c_{c-e s p, a} c_{d e n, a} \frac{c_{v o l, g}}{c_{\text {area }, s}} \frac{d X_{t, a}}{d t}=Q_{c v, c b-a}+Q_{c v, s s-a}+(7) \\
& +Q_{c v, c a l-a}-Q_{l t, t r p}-Q_{l t, t r p}-Q_{v e n}-Q_{\text {losses }}
\end{aligned}
$$

where $Q_{c v, c b-a}$ is the convective flux with the cover, $Q_{c v, s s-a}$ is the convective flux with the soil surface, $Q_{c v, c a l-a}$ is the convective flux with the heating pipes, $Q_{l, t r p}$ is the latent heat effect of the crop transpiration, $Q_{l t, e v p}$ is the latent heat effect of evaporation in the pools, $Q_{v e n t}$ is the heat lost by natural ventilation, $Q_{\text {losses }}$ is the heat lost by infiltration losses and $c_{c-s p, a}$ $c_{\text {den, } a}\left(c_{\text {vol,g}} / c_{\text {area,s }}\right)$ is the product of specific heat of air, air density and effective height of the greenhouse (greenhouse volume/soil surface area).

A model of humidity (water vapour content of the greenhouse air, $K g_{H 2 O} / K_{\text {air }}$ ) is based on a water mass balance equation. The main sources of vapour in a greenhouse are the crop transpiration, the evaporation of the soil surface and pools and the water influx by fogging or cooling. The vapour outflow takes place through the condensation on the internal side of the cover, the ventilation and the vapour lost by infiltration losses. The evaporation from the soil surface is neglected as it is mulched (Stanghellini, 1995). As artificial water influxes (cooling, fogging, etc.) were not installed, the mean water vapour content of the greenhouse air, $X_{\text {ha }}$, (absolute humidity) is modelled using the water mass balance equation given by equation (8).

$$
\begin{aligned}
& \frac{c_{\text {vol }, g}}{c_{\text {area }, \mathrm{s}}} c_{\text {den }, a} \frac{d X_{h, a}}{d t}=M_{\mathrm{H}_{2} \mathrm{O}, \mathrm{trp}}+M_{\mathrm{H}_{2} \mathrm{O}, \text { evp }}- \\
& -M_{\mathrm{H}_{2} \mathrm{O}, \text { cnd }}-M_{\mathrm{H}_{2} \mathrm{O}, \text { ven }}-M_{\mathrm{H}_{2} \mathrm{O}, \text { losses }}
\end{aligned}
$$

where $M_{H 2 O \text {,trp }}$ is the crop transpiration flux, $M_{H 2 O \text {,evp }}$ is the evaporation flux from the pools, $M_{H 2 O \text {, cnd }}$ is the condensation flux from the cover, $M_{H 2 O}$,ven is the outflow by natural ventilation and $M_{H 2 O}$,losses is the vapour lost by infiltration losses.

The values of the coefficients in previous equations have been obtained by using a large set of input/output data covering different operating conditions obtained at the real greenhouse and by iterative search in the range of values given by different authors using genetic algorithms. The previous equations are the basis for the formulation of a nonlinear simulation model using Dymola, as it is commented in the following section.

\section{MODELLING AND SIMULATION OF THE GREENHOUSE USING DYMOLA}

\subsection{Modelling of dynamical systems using Dymola}

Object-oriented modelling is a method for structuring models by applying the following ideas: use of declarative models, modularity, abstraction, encapsulation, information hiding, use of classes and inheritance. This methodology makes possible to describe models of many different domains like electrical circuits, mechanics, thermo-dynamics, etc. in an uniform way. A description of this methodology can be found in (Andersson, 1994).

In this work, the Dynamic Modelling Language Dymola (Elmqvist, 1978) has been used to model the greenhouse climate, taking advantage of the features of the object-oriented modelling approach. In 
addition to the modelling environment, Dymola implements automated formulae manipulation techniques such as: solving the causality assignment problem, generation of the equations that result from the couplings between different objects, automatic reduction of higher index systems and treatment of algebraic loops that often result from subsystem couplings and that also appear as a consequence of the reduction of higher index models. The motivation for using Dymola in this work is not due to the necessity of solving higher index problems (which is not a problem in this application), but that of using and object-oriented modelling language that allows the obtaining of noncausal descriptions of the subsystems components that form the final system to be simulated. The characteristics of object-oriented languages allow the development of models with reusing possibilities. In addition to the symbolic manipulations performed by Dymola over the whole system, it offers a rich repertory of ODE and DAE solvers that brings enough guaranties that the simulation has been performed correctly.

\subsection{Modelling of greenhouse climate}

The first stage of the modelling approach followed in this work has been to identify the model components and to define their interfaces (set of variables that connect the model with its environment). The behaviour of each component is described by DAE's, interconnected by their interfaces. Those models may be decomposed in submodels components allowing the use of hierarchical modelling techniques.

As has been mentioned in $\S 2$, the greenhouse climate dynamics may be decomposed in six submodels corresponding with the main state and output variables, interconnected via their interfaces. This methodology has many advantages, as helps to study each submodel independently and to debug it before connecting all of them to create the final compound model of the whole system. The submodels describe the PAR radiation, the inside air humidity and the temperature of the cover, the soil surface, the soil layer and the internal air. As will be mentioned in the following section, another advantage of the decomposition is that each submodel can be substituted by real measurements when these are available (e.g., measurements of the soil surface temperature).

The next subsections describe the classical model decomposition in interface and behaviour in objectoriented modelling of physical systems, in the case of the greenhouse climate model. For the sake of space, only the model of the internal air and the complete compound model are briefly described.

\section{The inside air temperature model}

口 Interface: formed by $X_{t s s}, U_{\text {heat }}, P_{\text {text }}, P_{\text {wsext }}$, $P_{\text {radsol,ext }}, U_{\text {vent }}, U_{\text {shad }}, X_{h_{-} i n v}, X_{t, c b}, P_{\text {white }}$ and $P_{L A I}$.

- Behaviour: described by ODE in equation (8).

The compound model: composed of the six mentioned submodels interconnected via their interface variables (Fig. 1).
- Interface: formed by $U_{\text {shad }}, P_{\text {radsol,ext }}, U_{\text {vent }}, P_{\text {text }}$, $P_{\text {wsex }}, P_{\text {habse }}, P_{t d s}, P_{\text {white }}$ and $P_{L A I}$.

a Behaviour: described by the equations that 'connect' the submodel components and the set of interface variables not connected between them to the compound model interface variables.

a Dymola code for the compound model: the list included in Fig. 2 contains a piece of code for the compound model, where the declaration of the submodels and their connection by different equations is shown.

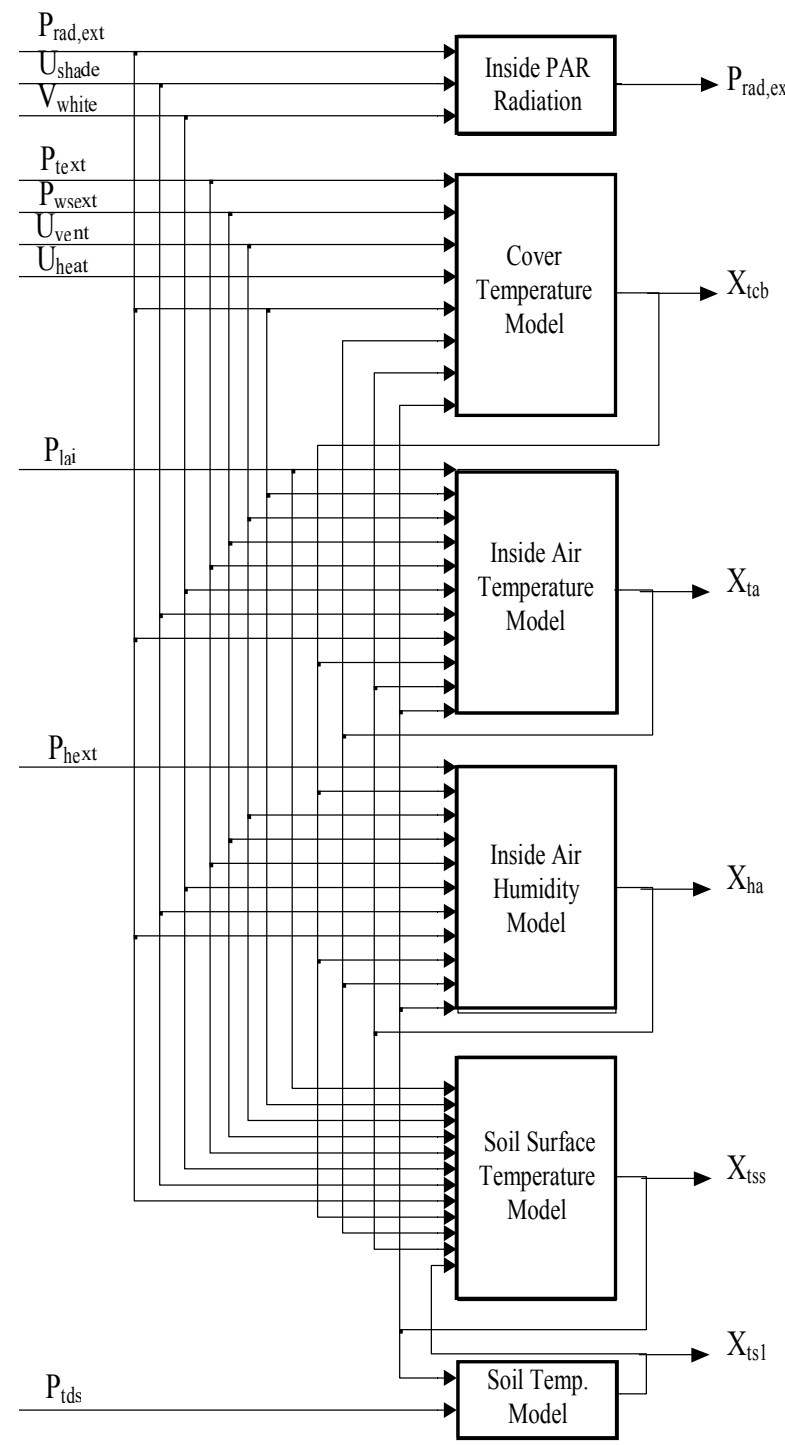

Fig. 1. Block diagram of the compound model

\section{RESULTS}

\subsection{Real greenhouse}

The experiments were carried out in an "Araba" greenhouse located in El Ejido (Almería, South-East Spain) near the sea (Fig. 3). It is a two symmetric curved slope roof with five North-South oriented

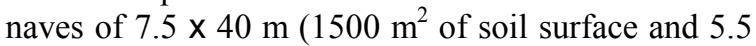
$\mathrm{m}$. high). The covering material is a PE film of 200 microns thick, laid on a structure made of galvanized steel. The control actuators are vents (lateral and roof), shade screen and hot water pipes heating. Several sensors were installed for data acquisition purposes. The soil temperature measurements were 
carried out through semiconductor sensors at different depths (both side of the mulching, immediately under the soil surface layer and at a depth of $50 \mathrm{~mm}$.). The greenhouse air temperature thermoresistent sensor and the air relative humidity capacitive sensor were placed at the top of the crop. Eight semiconductor contact sensors were installed on both cover sides to calculate the inside and outside cover temperatures on average. An external meteorological station was installed at $6 \mathrm{~m}$. height for acquiring measurements of temperature, relative humidity, solar and PAR radiation, wind speed and direction and rain. The system also acquires measurements of the actuators status. Data are sampled and stored each minute.

\subsection{Simulations}

To test the compound model with Dymola it is necessary to define a experiment in which all the interface variables from an instantiation of the greenhouse_compound model are assigned to experimental data registers, obtained from the data acquisition equipment installed at the greenhouse, as has been previously mentioned. Once the formulae manipulation has been performed by Dymola over the compound model, it generates a sequence of 115 equations (without algebraic loops), 5 of them ODE's and the rest are algebraic ones. This system of DAE's is discontinuous and these discontinuities are handled properly in Dymola by the definition in the submodels components of implicit state events. The selected interval of integration with DASSL is 13 days covering data obtained from the experimental greenhouse.

The first simulation shown in this section corresponds with the results obtained using the internal air submodel excited individually with data obtained from the experimental registers, and it is compared with the real temperature in order to validate the submodel. Fig. 4 represents both the simulated (Air_Xta) and real (Xta) internal air temperatures $\left({ }^{\circ} \overline{\mathrm{C}}\right)$.

The same procedure has been applied with the other output variables (cover temperature, soil temperature, etc.) in such a way that individual submodels have been simulated and validated. All these submodels can be integrated within the compound model, in such a way that the simulation of the greenhouse climate is a result of the interaction between the different submodels. Fig. 5 represents the evolution of the different output variables of the compound model, compared with the real values. Notice that the dynamic behaviour of the complete model is obtained by the interaction of simulated submodels connected properly. The variables in the plots are:

$\begin{array}{lcc}\text { Variable } & \underline{\text { Real }} & \text { Simulated } \\ \text { Cover temp. } & \text { Xtcb } & \text { Cover_Xtcb } \\ \text { Soil surface temp. } & \text { Xts } & \text { Soil_Xts } \\ \text { Soil layer 1 temp. } & \text { Xtsp } & \text { Soil_Xtsp } \\ \text { Inside air temp. } & \text { Xta } & \text { Air_Xta } \\ \text { Inside air hum. } & \text { Phabsi } & \text { Refi: : hume.XH_absa }\end{array}$

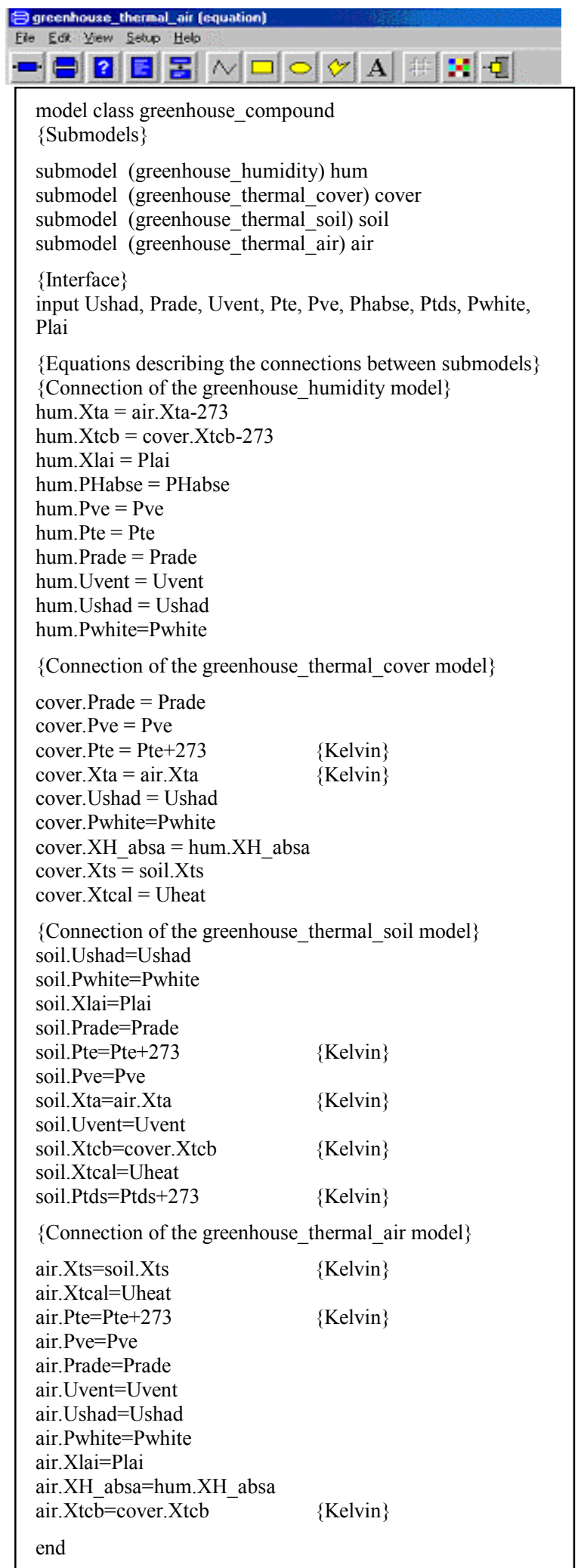

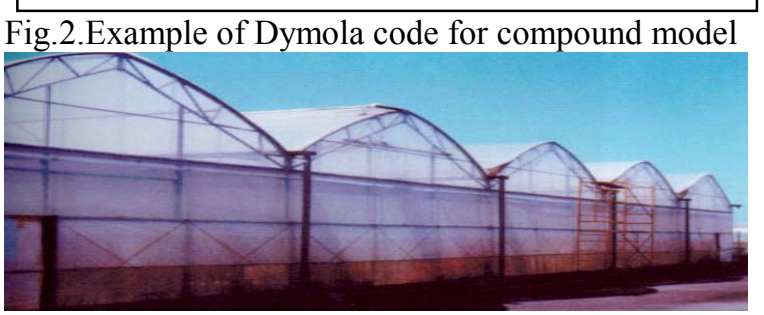

Fig. 3. Araba greenhouse

Table 2 provides some significant figures of the results obtained with both models, in terms of maximum (Max_abs), mean (Mean_abs) and standard deviation (std) absolute errors when compared with 
real data. Moreover, as can be seen from mean values, maximum errors tend to be spurious values when compared with general behaviour of the model. The model behaves quite well and constitutes an important tool in the analysis of the greenhouse dynamics and in the development of control schemes for optimising crop production. The methodology and way in which the model has been constructed allows it extension to other types of greenhouses.

\section{CONCLUSIONS}

The obtaining of adequate models for greenhouse climate modelling from mass and energy balances is a hard and time consuming task. Once the equations are adequately formulated, the use of modelling environments as Dymola and systematic procedures for decomposing the complete model in submodels, which can be independently validated, has shown to facilitate the implementation of the compound model (as an integration of the single submodels) and its extension to other types of greenhouses.

\section{ACKNOWLEDGEMENTS}

Authors would like to acknowledge CICYT for partially funding this work under grants QUI990663-C02-02 and DPI2001-2380-C02-02.

\section{REFERENCES}

Andersson, M. (1994). Object-Oriented Modeling and Simulation of Hybrid Systems. PhD thesis ISRN LUTFD2/TFRT-1043-SE, Dep. of Automatic Control, Lund Institute of Technology, Sweden.

Boisson, D. (1991). Etudes de la Climatisation de Serres par Ventilatión Naturalle en Periode Estivale: Modelisations et Simulations, Anex 7. PhD Thesis, Universite de Perpignan.

Brenan, K.E., S.L. Campbell, and L.R. Petzold (1989). Numerical Solution of Initial-Value Problems in Differential-Algebraic Equations. North-Holland.

Elmqvist, H. (1978). A Structured Model Language for Large Continuous Systems. PhD thesis TFRT-1015, Dept. of Automatic Control, Lund Institute of Technology, Sweden.

Garzoli, R.V., J. Blackwell (1981). An analysis of the Nocturnal Heat Loss from a Single Plastic Greenhouse. J. Agric. Engng. Res., 26, pp. 203-214.

Rodríguez, F., M.R. Arahal and M. Berenguel (1999). Application of Artificial NN for Greenhouse Climate Modelling. ECC99, Karlsruhe, Germany.

Rodríguez, F., M. Berenguel and A. Baille (2001). A Model of Greenhouse Crop Production. Part I: Model Development. Part II; Model Validation. Internal Report, Univ. Almería.

Stanghellini, C. (1987). Transpiration of greenhouse crops, IMAG Publication, Wageningen (Holland)

Stanghellini, C., de Jong, T. (1995). A model of humidity and its applications in a greenhouse. Agricultural and Forest Meteorology, 76, pp. 120-148.

Table 2. Comparative results

\begin{tabular}{lccccc}
\hline \multicolumn{7}{c}{ Air temp. submodel } \\
\hline Max_abs : 2.8002 & Mean_abs : 0.5006 & Std: & 0.5154 \\
\hline \multicolumn{7}{c}{ Compound model } \\
\hline Xta & Xha & Xtcb & Xtss & Xts 1 \\
\hline Max_abs & 6.1037 & 0.0079 & 5.1827 & 4.2122 & 1.3648 \\
\hline Mean_abs & 1.4418 & 0.001 & 0.8929 & 0.7003 & 0.3432 \\
\hline Std & 1.0913 & 0.0009625 & 0.7973 & 0.6323 & 0.2702 \\
\hline
\end{tabular}

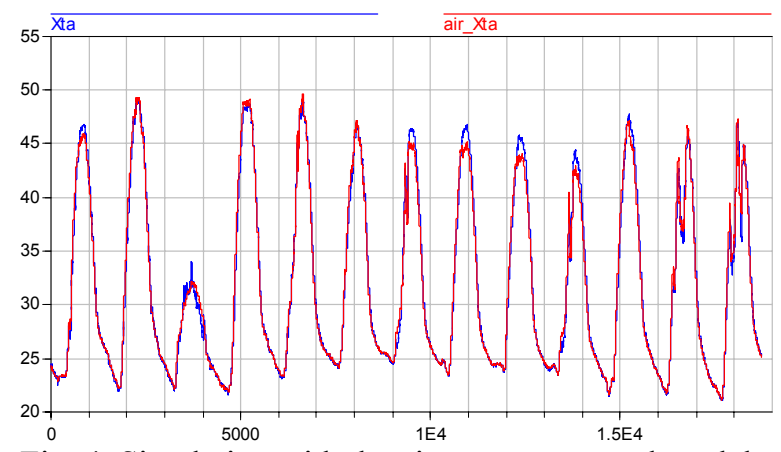

Fig. 4. Simulation with the air temperature submodel
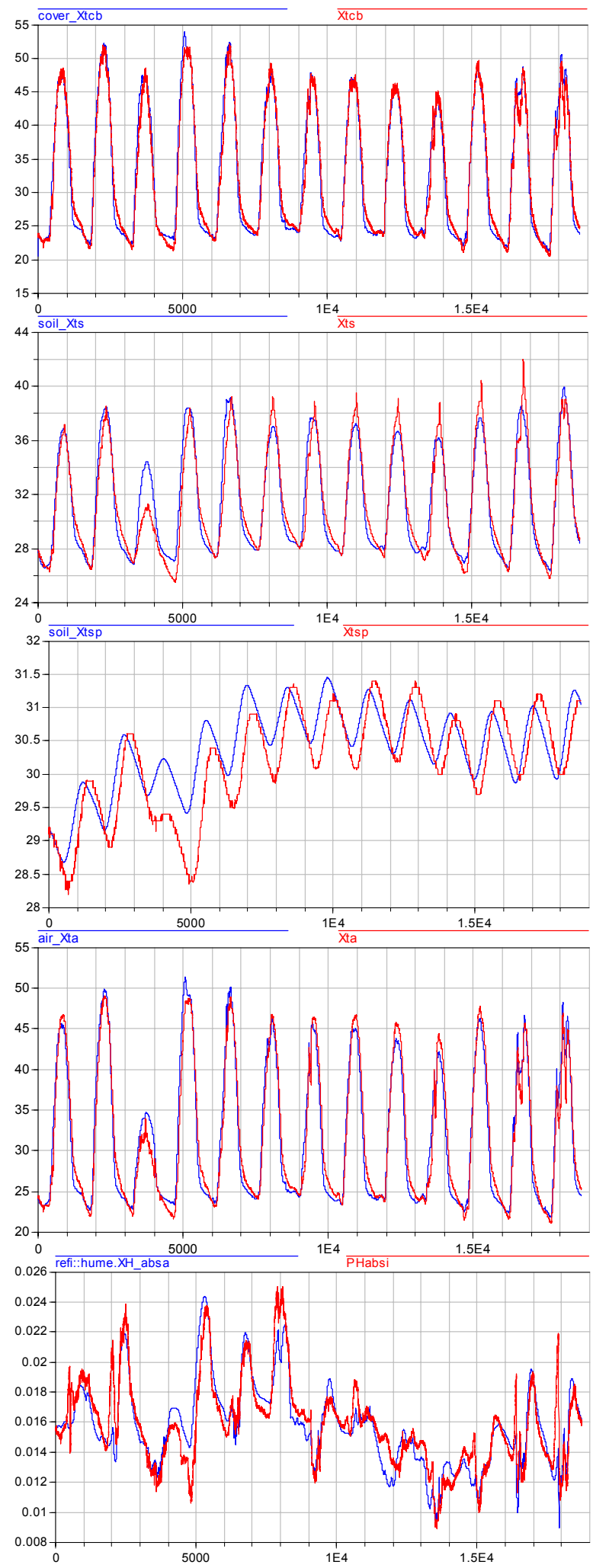

Fig. 5. Simulation results with the compound model 